\title{
Bell's Inequality Should Be Reconsidered in Quantum Language
}

\author{
Shiro Ishikawa \\ Department of Mathematics, Faculty of Science and Technology, Keio University, Yokohama, Japan \\ Email: ishikawa@math.keio.ac.jp
}

How to cite this paper: Ishikawa, S. (2017) Bell's Inequality Should Be Reconsidered in Quantum Language. Journal of Quantum Information Science, 7, 140-154. https://doi.org/10.4236/jqis.2017.74011

Received: October 27, 2017

Accepted: November 28, 2017

Published: December 1, 2017

Copyright $\odot 2017$ by author and Scientific Research Publishing Inc. This work is licensed under the Creative Commons Attribution International License (CC BY 4.0).

http://creativecommons.org/licenses/by/4.0/

\begin{abstract}
Bell's inequality itself is usually considered to belong to mathematics and not quantum mechanics. We think that this is making our understanding of Bell' theory be confused. Thus in this paper, contrary to Bell's spirit (which inherits Einstein's spirit), we try to discuss Bell's inequality in the framework of quantum theory with the linguistic Copenhagen interpretation. And we clarify that the violation of Bell's inequality (i.e., whether or not Bell's inequality holds) does not depend on whether classical systems or quantum systems, but depend on whether a combined measurement exists or not. And further we conclude that our argument (based on the linguistic Copenhagen interpretation) should be regarded as a scientific representation of Bell's philosophical argument (based on Einstein's spirit).
\end{abstract}

\section{Keywords}

Bohr-Einstein Debates, Bell's Inequality, Combined Observable, Linguistic Copenhagen Interpretation, Quantum Language

\section{Review: Quantum Language (=Measurement Theory (=MT))}

\subsection{Introduction}

Recently (cf. refs. [1]-[10], also see $\left(\mathrm{B}_{0}\right)-\left(\mathrm{B}_{3}\right)$ later), we proposed quantum language, which was not only characterized as the metaphysical and linguistic turn of quantum mechanics but also the linguistic turn of dualistic idealism. And further we believe that quantum language should be regarded as the foundations of quantum information science. Quantum language is formulated as follows.

(A) $\frac{\text { Quantumlanguage }}{\text { (language) }}=\frac{\text { Measurement }}{\text { (Axiom 1) }}+\underset{\text { (Axiom 2) }}{\text { Causality }}+\frac{\text { Linguistic(Copenhagen)interpretation }}{\text { (how to use Axioms 1 and 2) }}$

Note that this theory (A) is not physics but a kind of language based on the 
quantum mechanical world view. That is, we think that the location of quantum language in the history of world-descriptions is as follows.

And in Figure 1, we think that the following four are equivalent (refs. [1] [8]):

$\left(\mathrm{B}_{0}\right)$ to propose quantum language ( $c f$. $(10)$ in Figure 1, ref. [1] [8]).

$\left(B_{1}\right)$ to clarify the Copenhagen interpretation of quantum mechanics ( $c f$. (7) in Figure 1, refs. [2] [7] [11]), that is, the linguistic Copenhagen interpretation is the true figure of so-called Copenhagen interpretation.

$\left(\mathrm{B}_{2}\right)$ to clarify the final goal of the dualistic idealism (cf. 8) in Figure 1, refs. [3] [9]).

$\left(\mathrm{B}_{3}\right)$ to reconstruct statistics in the dualistic idealism (cf. (9) in Figure 1, refs. [4] [5] [6] [12]).

In Bohr-Einstein debates (refs. [13] [14]), Einstein's standing-point (that is, "the moon is there whether one looks at it or not" (i.e., physics holds without observers)) is on the side of the realistic world view in Figure 1. On the other hand, we think that Bohr's standing point (that is, "to be is to be perceived" (i.e., there is no science without measurements)) is on the side of the linguistic world view in Figure 1 (though N. Bohr might believe that the Copenhagen interpretation (proposed by his school) belongs to physics).

In this paper, contrary to Bell's spirit (which inherits Einstein's spirit), we try to discuss Bell's inequality (refs. [15] [16] [17] [18]) in quantum language (i.e., quantum theory with the linguistic Copenhagen interpretation). And we clarify that whether or not Bell's inequality holds does not depend on whether classical systems or quantum systems (in Section 3), but depend on whether a combined measurement exists or not (in Section 2). And further we assert that our argument (based on the linguistic Copenhagen interpretation) should be regarded as a scientific representation of Bell's philosophical argument (based on Einstein's spirit).

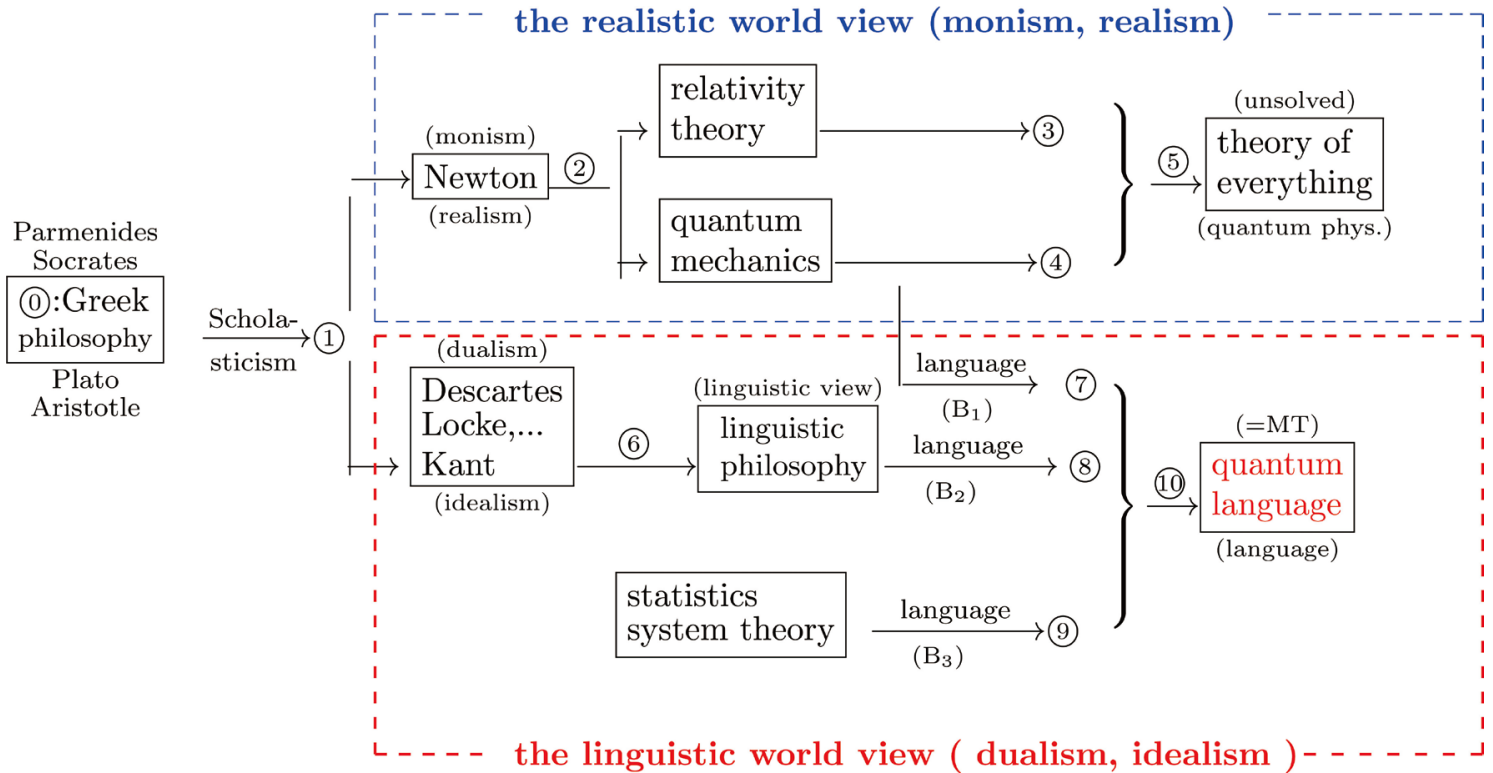

Figure 1. The history of the world-descriptions. 


\subsection{Quantum Language (=Measurement Theory); Mathematical Preparations}

Now we shall explain the measurement theory (A).

Consider an operator algebra $B(H)$ (i.e., an operator algebra composed of all bounded linear operators on a Hilbert space $H$ with the norm $\left.\|F\|_{B(H)}=\sup _{\|u\|_{H}=1}\|F u\|_{H}\right)$, and consider the pair $[\mathcal{A}, \mathcal{N}]_{B(H)}$, called a basic structure. Here, $\mathcal{A}(\subseteq B(H))$ is a $C$-algebra, and $\mathcal{N}(\mathcal{A} \subseteq \mathcal{N} \subseteq B(H))$ is a particular $C$-algebra (called a $W^{*}$-algebra) such that $\mathcal{N}$ is the weak closure of $\mathcal{A}$ in $B(H)$.

The measurement theory (=quantum language) is classified as follows. measurement theory $(\mathrm{A})$

(C) $= \begin{cases}\left(\mathrm{C}_{1}\right) \text { : quantum system theory } & (\text { when } \mathcal{A}=\mathcal{C}(H)) \\ \left(\mathrm{C}_{2}\right): \text { classical system theory } & \left(\text { when } \mathcal{A}=C_{0}(\Omega)\right)\end{cases}$

That is, when $\mathcal{A}=\mathcal{C}(H)$, the $C$-algebra composed of all compact operators on a Hilbert space $H$, the $\left(\mathrm{C}_{1}\right)$ is called quantum measurement theory (or, quantum system theory), which can be regarded as the linguistic aspect of quantum mechanics. Also, when $\mathcal{A}$ is commutative (that is, when $\mathcal{A}$ is characterized by $C_{0}(\Omega)$, the $C$-algebra composed of all continuous complex-valued functions vanishing at infinity on a locally compact Hausdorff space $\Omega$ (cf. [19] [20])), the $\left(\mathrm{C}_{2}\right)$ is called classical measurement theory (or, classical system theory).

Also, note (cf. [19]) that, when $\mathcal{A}=\mathcal{C}(H)$,

1) $\mathcal{A}^{*}=\operatorname{Tr}(H)(=$ trace class $), \quad \mathcal{N}=B(H), \quad \mathcal{N}_{*}=\operatorname{Tr}(H) \quad$ (i.e., pre-dual space).

Also, when $\mathcal{A}=C_{0}(\Omega)$,

2) $\mathcal{A}^{*}=$ "the space of all signed measures on $\Omega$ ", $\mathcal{N}=L^{\infty}(\Omega, v)\left(\subseteq B\left(L^{2}(\Omega, v)\right)\right), \quad \mathcal{N}_{*}=L^{1}(\Omega, v)$, where $v$ is some measure on $\Omega$ (cf. [19]). Also, the $L^{\infty}(\Omega, v)$ is usually denoted by $L^{\infty}(\Omega)$.

Let $\mathcal{A}(\subseteq B(H))$ be a $C$-algebra, and let $\mathcal{A}^{*}$ be the dual Banach space of $\mathcal{A}$. That is, $\mathcal{A}^{*}=\{\rho \mid \rho$ is a continuous linear functional on $\mathcal{A}\}$, and the norm $\|\rho\|_{\mathcal{A}^{*}}$ is defined by $\sup \left\{|\rho(F)| \mid F \in \mathcal{A}\right.$ such that $\left.\|F\|_{\mathcal{A}}\left(=\|F\|_{B(H)}\right) \leq 1\right\}$. Define the mixed state $\rho\left(\in \mathcal{A}^{*}\right)$ such that $\|\rho\|_{\mathcal{A}^{*}}=1$ and $\rho(F) \geq 0$ for all $F \in \mathcal{A}$ such that $F \geq 0$. And define the mixed state space $\mathfrak{S}^{m}\left(\mathcal{A}^{*}\right)$ such that

$$
\mathfrak{S}^{m}\left(\mathcal{A}^{*}\right)=\left\{\rho \in \mathcal{A}^{*} \mid \rho \text { is a mixed state }\right\} .
$$

A mixed state $\rho\left(\in \mathfrak{S}^{m}\left(\mathcal{A}^{*}\right)\right)$ is called a pure state if it satisfies that $\rho=\theta \rho_{1}+(1-\theta) \rho_{2}$ for some $\rho_{1}, \rho_{2} \in \mathfrak{S}^{m}\left(\mathcal{A}^{*}\right)$ and $0<\theta<1$ implies $\rho=\rho_{1}$ $=\rho_{2}$. Put

$$
\mathfrak{S}^{p}\left(\mathcal{A}^{*}\right)=\left\{\rho \in \mathfrak{S}^{m}\left(\mathcal{A}^{*}\right) \mid \rho \text { is a pure state }\right\},
$$

which is called a state space. It is well known (cf. [19]) that $\mathfrak{S}^{p}\left(\mathcal{C}(H)^{*}\right)=\{|u\rangle\langle u|$ 
(i.e., the Dirac notation) $\left.\mid\|u\|_{H}=1\right\}$, and $\mathfrak{S}^{p}\left(C_{0}(\Omega)^{*}\right)=\left\{\delta_{\omega_{0}} \mid \delta_{\omega_{0}}\right.$ is a point measure at $\left.\omega_{0} \in \Omega\right\}$, where $\int_{\Omega} f(\omega) \delta_{\omega_{0}}(\mathrm{~d} \omega)=f\left(\omega_{0}\right) \quad\left(\forall f \in C_{0}(\Omega)\right)$. The latter implies that $\mathfrak{S}^{p}\left(C_{0}(\Omega)^{*}\right)$ can be also identified with $\Omega$ (called a spectrum space or simply spectrum) such as

$$
\mathfrak{S}^{p}\left(C_{0}(\Omega)^{*}\right) \ni \delta_{\omega} \leftrightarrow \omega \in \underset{\text { (states pace) }}{\Omega}
$$

For instance, in the above 2) we must clarify the meaning of the "value" of $F\left(\omega_{0}\right)$ for $F \in L^{\infty}(\Omega, v)$ and $\omega_{0} \in \Omega$. An element $F(\in \mathcal{N})$ is said to be essentially continuous at $\rho_{0}\left(\in \mathfrak{S}^{p}\left(\mathcal{A}^{*}\right)\right)$, if there uniquely exists a complex number $\alpha$ such that

- if $\rho\left(\in \mathcal{N}_{*},\|\rho\|_{\mathcal{N}_{*}}=1\right)$ converges to $\rho_{0}\left(\in \mathfrak{S}^{p}\left(\mathcal{A}^{*}\right)\right)$ in the sense of weak ${ }^{*}$ topology of $\mathcal{A}^{*}$, that is,

$$
\rho(G) \rightarrow \rho_{0}(G)(\forall G \in \mathcal{A}(\subseteq \mathcal{N})),
$$

then $\rho(F)$ converges to $\alpha$.

And the value of $\rho_{0}(F)$ is defined by the $\alpha$.

According to the noted idea (cf. [21]), an observable $\mathrm{O}:=(X, \mathcal{F}, F)$ in $\mathcal{N}$ is defined as follows:

1) [ $\sigma$-field] $X$ is a set, $\mathcal{F}\left(\subseteq 2^{X}=\mathcal{P}(X)\right.$, the power set of $\left.X\right)$ is a $\sigma$-field of $X$, that is, " $\Xi_{1}, \Xi_{2}, \cdots \in \mathcal{F} \Rightarrow \bigcup_{n=1}^{\infty} \Xi_{n} \in \mathcal{F}$ ”, “ $\Xi \in \mathcal{F} \Rightarrow X \backslash \Xi \in \mathcal{F}$ ”, “ $X \in \mathcal{F}$ ".

2) [Countable additivity] $F$ is a mapping from $\mathcal{F}$ to $\mathcal{N}$ satisfying: a) for every $\Xi \in \mathcal{F}, F(\Xi)$ is a non-negative element in $\mathcal{N}$ such that $0 \leq F(\Xi) \leq I$, b) $F(\varnothing)=0$ and $F(X)=I$, where 0 and $I$ is the 0 -element and the identity in $\mathcal{N}$ respectively. (c): for any countable decomposition $\left\{\Xi_{1}, \Xi_{2}, \cdots, \Xi_{n}, \cdots\right\}$ of $\Xi$ (i.e., $\left.\Xi, \Xi_{n} \in \mathcal{F}(n=1,2,3, \cdots), \quad \bigcup_{n=1}^{\infty} \Xi_{n}=\Xi, \quad \Xi_{i} \cap \Xi_{j}=\varnothing(i \neq j)\right)$, it holds that $F(\Xi)=\sum_{n=1}^{\infty} F\left(\Xi_{n}\right)$ in the sense of weak topology in $\mathcal{N}$.

Remark 1. Quantum language has two formulations (i.e., the $C$-algebraic formulation and the $W$-algebraic formulation). In this paper, we devote ourselves to the $W^{*}$-algebraic formulation, which may, from the mathematical point of view, be superiority to the $C$-algebraic formulation. That is, in the above 2), the countable additivity (i.e., $F(\Xi)=\lim _{N \rightarrow \infty} \sum_{n=1}^{N} F\left(\Xi_{n}\right)$ ) is naturally discussed in the $W$-algebraic formulation. However, the $C$-algebraic formulation has a merit such that we can use it without sufficient mathematical preparation. For the $C$-algebraic version of this paper, see my preprint [10].

\subsection{Axiom 1 [Measurement] and Axiom 2 [Causality]}

With any system $S$, a basic structure $[\mathcal{A}, \mathcal{N}]_{B(H)}$ can be associated in which the measurement theory (A) of that system can be formulated. A state of the system $S$ is represented by an element $\rho\left(\in \mathfrak{S}^{p}\left(\mathcal{A}^{*}\right)\right)$ and an observable is represented by an observable $\mathrm{O}:=(X, \mathcal{F}, F)$ in $\mathcal{N}$. Also, the measurement of the observable $\mathrm{O}$ for the system $S$ with the state $\rho$ is denoted by $\mathrm{M}_{\mathcal{N}}\left(\mathrm{O}, S_{[\rho]}\right)$ (or more precisely, $\left.\mathrm{M}_{\mathcal{N}}\left(\mathrm{O}:=(X, \mathcal{F}, F), S_{[\rho]}\right)\right)$. An observer can obtain a measured value 
$x(\in X)$ by the measurement $\mathrm{M}_{\mathcal{N}}\left(\mathrm{O}, S_{[\rho]}\right)$.

The Axiom 1 presented below is a kind of mathematical generalization of Born's probabilistic interpretation of quantum mechanics (cf. ref. [22]). And thus, it is a statement without reality.

Now we can present Axiom 1 in the $W^{*}$-algebraic formulation as follows.

Axiom 1 [ Measurement ]. The probability that a measured value $x \quad(\in X)$ obtained by the measurement $\mathrm{M}_{\mathcal{N}}\left(\mathrm{O}:=(X, \mathcal{F}, F), S_{[\rho]}\right)$ belongs to a set $\Xi(\in \mathcal{F})$ is given by $\rho(F(\Xi))$ if $F(\Xi)$ is essentially continuous at $\rho\left(\in \mathfrak{S}^{p}\left(\mathcal{A}^{*}\right)\right)$.

Next, we explain Axiom 2. Let $\left[\mathcal{A}_{1}, \mathcal{N}_{1}\right]_{B\left(H_{1}\right)}$ and $\left[\mathcal{A}_{2}, \mathcal{N}_{2}\right]_{B\left(H_{2}\right)}$ be basic structures. A continuous linear operator $\Phi_{1,2}: \mathcal{N}_{2}$ (with weak topology) $\rightarrow \mathcal{N}_{1}$ (with weak topology) is called a Markov operator, if it satisfies that 1) $\Phi_{1,2}\left(F_{2}\right) \geq 0$ for any non-negative element $F_{2}$ in $\left.\mathcal{N}_{2}, 2\right) \quad \Phi_{1,2}\left(I_{2}\right)=I_{1}$, where $I_{k}$ is the identity in $\mathcal{N}_{k},(k=1,2)$. In addition to the above 1) and 2), in this paper we assume that $\Phi_{1,2}\left(\mathcal{A}_{2}\right) \subseteq \mathcal{A}_{1}$ and $\sup \left\{\left\|\Phi_{1,2}\left(F_{2}\right)\right\|_{\mathcal{A}_{1}} \mid F_{2} \in \mathcal{A}_{2}\right.$ such that $\left.\left\|F_{2}\right\|_{\mathcal{A}_{2}} \leq 1\right\}=1$.

It is clear that the dual operator $\Phi_{1,2}^{*}: \mathcal{A}_{1}^{*} \rightarrow \mathcal{A}_{2}^{*}$ satisfies that $\Phi_{1,2}^{*}\left(\mathfrak{S}^{m}\left(\mathcal{A}_{1}^{*}\right)\right)$ $\subseteq \mathfrak{S}^{m}\left(\mathcal{A}_{2}^{*}\right)$. If it holds that $\Phi_{1,2}^{*}\left(\mathfrak{S}^{p}\left(\mathcal{A}_{1}^{*}\right)\right) \subseteq \mathfrak{S}^{p}\left(\mathcal{A}_{2}^{*}\right)$, the $\Phi_{1,2}$ is said to be deterministic. If it is not deterministic, it is said to be non-deterministic or decoherence. Here note that, for any observable $\mathrm{O}_{2}:=\left(X, \mathcal{F}, F_{2}\right)$ in $\mathcal{N}_{2}$, the $\left(X, \mathcal{F}, \Phi_{1,2} F_{2}\right)$ is an observable in $\mathcal{N}_{1}$.

Now Axiom 2 in the measurement theory (A) is presented as follows:

Axiom 2 [Causality]. Let $t_{1} \leq t_{2}$. The causality is represented by a Markov operator $\Phi_{t_{1}, t_{2}}: N_{t_{2}} \rightarrow N_{t_{1}}$.

\subsection{The Linguistic Interpretation (=The Manual to Use Axioms 1 and 2)}

In the above, Axioms 1 and 2 are kinds of spells, (i.e., incantation, magic words, metaphysical statements), and thus, it is nonsense to verify them experimentally. Therefore, what we should do is not "to understand" but "to use". After learning Axioms 1 and 2 by rote, we have to improve how to use them through trial and error.

We can do well even if we do not know the linguistic interpretation. However, it is better to know the linguistic interpretation (=the manual to use Axioms 1 and 2), if we would like to make progress quantum language early.

The essence of the manual is as follows:

(D) Only one measurement is permitted. And thus, the state after a measurement is meaningless since it cannot be measured any longer. Thus, the collapse of the wavefunction is prohibited (cf. [7]). We are not concerned with anything after measurement. That is, any statement including the phrase after the measurement is wrong. Also, the causality should be assumed only in the side of system, however, a state never moves. Thus, the Heisenberg picture should be adopted, and thus, the Schrödinger picture should be prohibited. Also, it is added that there is no probability without a measurement.

and so on. For details, see [8]. 


\subsection{Generalized Simultaneous Measurement, Parallel Measurement}

Definition 2. [Generalized simultaneous observable, Generalized simultaneous measurement $]$ Let $[\mathcal{A}, \mathcal{N}]_{B(H)}$ be a basic structure. Consider observables $\mathrm{O}_{k}=\left(X_{k}, \mathcal{F}_{k}, F_{k}\right) \quad(k=1,2, \cdots, K)$ in $\mathcal{N}$. Let $\left(x_{k=1}^{K} X_{k}, \mathbb{\bigotimes}_{k=1}^{K} \mathcal{F}_{k}\right)$ be the product measurable space, i.e., the product space $\times_{k=1}^{K} X_{k}$ and the product $\sigma$-field $\bigotimes_{k=1}^{K} \mathcal{F}_{k}$, which is defined by the smallest $\sigma$-field that contains a family $\left\{x_{k=1}^{K} \Xi_{k} \mid \Xi_{k} \in \mathcal{F}_{k}, k=1,2, \cdots, K\right\}$. An observable $\mathrm{O}={ }_{\mathrm{q}_{k=1,2, \cdots, K}} \mathrm{O}_{k}=\left(\times_{k=1}^{K} X_{k}, \otimes_{k=1}^{K} \mathcal{F}_{k}, F\right)$ in $\mathcal{N}$ is called the generalized simultaneous observable (or, quasi-product observable, combined observable, etc.) of $\mathrm{O}_{k}$ $(k=1,2, \cdots, K)$, if it holds that

$$
\begin{aligned}
& F\left(X_{1} \times X_{2} \times \cdots \times X_{k-1} \times \Xi_{k} \times X_{k+1} \times \cdots \times X_{K}\right) \\
& =F_{k}\left(\Xi_{k}\right),\left(\forall \Xi_{k} \in \mathcal{F}_{k}, k=1,2, \cdots, K\right)
\end{aligned}
$$

Also, the measurement $\mathrm{M}_{\mathcal{N}}\left(\mathrm{O}, S_{\left[\rho_{0}\right]}\right)$ is called a generalized simultaneous measurement of measurements $\mathrm{M}_{\mathcal{N}}\left(\mathrm{O}_{k}, S_{\left[\rho_{0}\right]}\right)(k=1,2, \cdots, K)$. A generalized simultaneous observable is called a simultaneous observable, if it holds:

$$
\begin{aligned}
& F\left(\Xi_{1} \times \Xi_{2} \times \cdots \times \Xi_{k-1} \times \Xi_{k} \times \Xi_{k+1} \times \cdots \times \Xi_{K}\right) \\
& =\underset{k=1}{K} F_{k}\left(\Xi_{k}\right),\left(\forall \Xi_{k} \in \mathcal{F}_{k}, k=1,2, \cdots, K\right)
\end{aligned}
$$

Note that the existence and the uniqueness of a generalized simultaneous observable $\mathrm{O}=\left(\times_{k=1}^{K} X_{k}, \bigotimes_{k=1}^{K} \mathcal{F}_{k}, F\right)$ in $\mathcal{N}$ are not assured in general, however the simultaneous observable always exists if observables $\mathrm{O}_{k}(k=1,2, \cdots, K)$ commute, i.e.,

$$
F_{k}\left(\Xi_{k}\right) F_{l}\left(\Xi_{l}\right)=F_{l}\left(\Xi_{l}\right) F_{k}\left(\Xi_{k}\right),\left(\forall \Xi_{k} \in \mathcal{F}_{k}, \forall \Xi_{l} \in \mathcal{F}_{l}, k \neq l\right)
$$

Definition 3. [Parallel observable, Parallel measurement] For each $k=1,2, \cdots, K$, consider a basic structure $\left[\mathcal{A}_{k}, \mathcal{N}_{k}\right]_{B\left(H_{k}\right)}$ and a measurement $\mathrm{M}_{\mathcal{N}_{k}}\left(\mathrm{O}_{k}:=\left(X_{k}, \mathcal{F}_{k}, F_{k}\right), S_{\left[\rho_{k}\right]}\right)$. We consider the spatial tensor $W^{*}$-algebra $\otimes_{k=1}^{K} \mathcal{N}_{k}\left(\subseteq B\left(\otimes_{k=1}^{K} H_{k}\right)\right)$, and consider the product measurable space $\left(\times_{k=1}^{K} X_{k}, \bigotimes_{k=1}^{K} \mathcal{F}_{k}\right)$. Consider the observable $\otimes_{k=1}^{K} \mathrm{O}_{k}=\left(x_{k=1}^{K} X_{k}, \bigotimes_{k=1}^{K} \mathcal{F}_{k}, \tilde{F}\right)$ in $\bigotimes_{k=1}^{K} \mathcal{N}_{k}$ such that

$$
\tilde{F}\left(x_{k=1}^{K} \Xi_{k}\right)=\otimes_{k=1}^{K} F_{k}\left(\Xi_{k}\right)\left(\forall \Xi_{k} \in \mathcal{F}_{k}, k=1,2, \cdots, K\right) .
$$

which is called the parallel observable of $\mathrm{O}_{k}:=\left(X_{k}, \mathcal{F}_{k}, F_{k}\right) \quad(k=1,2, \cdots, K)$. And let $\otimes_{k=1}^{K} \rho_{k} \in \mathfrak{S}^{p}\left(\left(\bigotimes_{k=1}^{K} \mathcal{A}_{k}\right)^{*}\right)$. Then the measurement $\mathrm{M}_{\otimes_{k=1}^{K} \mathcal{N}_{k}}$ $\left(\otimes_{k=1}^{K} \mathrm{O}_{k}=\left(\times_{k=1}^{K} X_{k}, \otimes_{k=1}^{K} \mathcal{F}_{k}, \bigotimes_{k=1}^{K} F_{k}\right), S_{\left[\otimes_{k=1}^{K} \rho_{k}\right]}\right)$ (which is also denoted by $\left.\otimes_{k=1}^{K} \mathrm{M}_{\mathcal{N}_{k}}\left(\mathrm{O}_{k}, S_{\left[\rho_{k}\right]}\right)\right)$ is called a parallel measurement of $\mathrm{M}_{\mathcal{N}_{k}}\left(\mathrm{O}_{k}=\left(X_{k}, \mathcal{F}_{k}, F_{k}\right), S_{\left[\rho_{k}\right]}\right) \quad(k=1,2, \cdots, K)$. Note that the parallel measurement always exists uniquely. 


\section{Bell's Inequality Always Holds in Classical and Quantum Systems}

\section{Our Main Assertion about Bell's Inequality}

In this paper, I assert that Bell's inequality should be studied in the framework of quantum language (i.e., quantum theory with the linguistic Copenhagen interpretation). Let us start from the following definition, which is a slight modification of the generalized simultaneous observable in Definition 2. That is, Definitions $2-4$ are due to the linguistic Copenhagen interpretation (D), "Only one measurement is permitted".

Definition 4 [Combined observable (cf. ref. [12])] Let $[\mathcal{A}, \mathcal{N}]_{B(H)}$ be a basic structure. Put $X=\{-1,1\}$. Consider four observables: $\mathrm{O}_{13}=\left(X^{2}, \mathcal{P}\left(X^{2}\right), F_{13}\right)$, $\mathrm{O}_{14}=\left(X^{2}, \mathcal{P}\left(X^{2}\right), F_{14}\right), \quad \mathrm{O}_{23}=\left(X^{2}, \mathcal{P}\left(X^{2}\right), F_{23}\right), \quad \mathrm{O}_{24}=\left(X^{2}, \mathcal{P}\left(X^{2}\right), F_{24}\right)$ in $\mathcal{N}$. The four observables are said to be combinable if there exists an observable $\mathrm{O}=\left(X^{4}, \mathcal{P}\left(X^{4}\right), F\right)$ in $\mathcal{N}$ such that

$$
\begin{aligned}
& F_{13}\left(\left\{\left(x_{1}, x_{3}\right)\right\}\right)=F\left(\left\{x_{1}\right\} \times X \times\left\{x_{3}\right\} \times X\right), \\
& F_{14}\left(\left\{\left(x_{1}, x_{4}\right)\right\}\right)=F\left(\left\{x_{1}\right\} \times X \times X \times\left\{x_{4}\right\}\right), \\
& F_{23}\left(\left\{\left(x_{2}, x_{3}\right)\right\}\right)=F\left(X \times\left\{x_{2}\right\} \times\left\{x_{3}\right\} \times X\right), \\
& F_{24}\left(\left\{\left(x_{2}, x_{4}\right)\right\}\right)=F\left(X \times\left\{x_{2}\right\} \times X \times\left\{x_{4}\right\}\right)
\end{aligned}
$$

for any $\left(x_{1}, x_{2}, x_{3}, x_{4}\right) \in X^{4}$. The observable $\mathrm{O}$ is said to be a combined observable of $\mathrm{O}_{i j} \quad(i=1,2, j=3,4)$. Also, the measurement $\mathrm{M}_{\mathcal{N}}\left(\mathrm{O}=\left(X^{4}, \mathcal{P}\left(X^{4}\right), F\right), S_{\left[\rho_{0}\right]}\right)$ is called the combined measurement of $\mathrm{M}_{\mathcal{N}}\left(\mathrm{O}_{13}, S_{\left[\rho_{0}\right]}\right), \mathrm{M}_{\mathcal{N}}\left(\mathrm{O}_{14}, S_{\left[\rho_{0}\right]}\right), \mathrm{M}_{\mathcal{N}}\left(\mathrm{O}_{23}, S_{\left[\rho_{0}\right]}\right)$ and $\mathrm{M}_{\mathcal{N}}\left(\mathrm{O}_{24}, S_{\left[\rho_{0}\right]}\right)$.

Remark 5. 1) Note that the Formula (4) implies that

$$
\begin{array}{ll}
F_{13}(\{x\} \times X)=F_{14}(\{x\} \times X), & F_{23}(\{x\} \times X)=F_{24}(\{x\} \times X), \\
F_{13}(X \times\{x\})=F_{23}(X \times\{x\}), & F_{14}(X \times\{x\})=F_{24}(X \times\{x\}),
\end{array}
$$

for all $x \in X$.

2) Syllogism (i.e., $[[A \Rightarrow B] \wedge[B \Rightarrow C]] \Rightarrow[A \Rightarrow C]$ ) does not hold in quantum systems but in classical systems (cf. ref. [8]). A certain combined observable plays an important role in the proof of the classical syllogism ( $c f$. ref. [12]).

The following theorem is all of our insistence concerning Bell's inequality. We assert that this is the true Bell's inequality.

Theorem 6. [Bell's inequality in quantum language] Let $[\mathcal{A}, \mathcal{N}]_{B(H)}$ be a basic structure. Put $X=\{-1,1\}$. Fix the pure state $\rho_{0}\left(\in \mathfrak{S}^{p}\left(\mathcal{A}^{*}\right)\right)$. And consider the four measurements $\mathrm{M}_{\mathcal{N}}\left(\mathrm{O}_{13}=\left(X^{2}, \mathcal{P}\left(X^{2}\right), F_{13}\right), S_{\left[\rho_{0}\right]}\right)$,

$\mathrm{M}_{\mathcal{N}}\left(\mathrm{O}_{14}=\left(X^{2}, \mathcal{P}\left(X^{2}\right), F_{14}\right), S_{\left[\rho_{0}\right]}\right), \quad \mathrm{M}_{\mathcal{N}}\left(\mathrm{O}_{23}=\left(X^{2}, \mathcal{P}\left(X^{2}\right), F_{23}\right), S_{\left[\rho_{0}\right]}\right) \quad$ and $\mathrm{M}_{\mathcal{N}}\left(\mathrm{O}_{24}=\left(X^{2}, \mathcal{P}\left(X^{2}\right), F_{24}\right), S_{\left[\rho_{0}\right]}\right)$. Or equivalently, consider the parallel measurement $\otimes_{i=1,2, j=3,4} \mathrm{M}_{\mathcal{N}}\left(\mathrm{O}_{i j}=\left(X^{2}, \mathcal{P}\left(X^{2}\right), F_{i j}\right), S_{\left[\rho_{0}\right]}\right)$. Define four correlation functions $(i=1,2, j=3,4)$ such that 


$$
R_{i j}=\sum_{(u, v) \in X \times X} u \cdot v \rho_{0}\left(F_{i j}(\{(u, v)\})\right)
$$

Assume that four observables $\mathrm{O}_{13}=\left(X^{2}, \mathcal{P}\left(X^{2}\right), F_{13}\right), \mathrm{O}_{14}=\left(X^{2}, \mathcal{P}\left(X^{2}\right), F_{14}\right)$, $\mathrm{O}_{23}=\left(X^{2}, \mathcal{P}\left(X^{2}\right), F_{23}\right)$ and $\mathrm{O}_{24}=\left(X^{2}, \mathcal{P}\left(X^{2}\right), F_{24}\right)$ are combinable, that is, we have the combined observable $\mathrm{O}=\left(X^{4}, \mathcal{P}\left(X^{4}\right), F\right)$ in $\mathcal{N}$ such that it satisfies (4). Then we have a combined measurement $\mathrm{M}_{\mathcal{N}}\left(\mathrm{O}=\left(X^{4}, \mathcal{P}\left(X^{4}\right), F\right), S_{\left[\rho_{0}\right]}\right)$ of $\mathbf{M}_{\mathcal{N}}\left(\mathrm{O}_{13}, S_{\left[\rho_{0}\right]}\right), \quad \mathbf{M}_{\mathcal{N}}\left(\mathrm{O}_{14}, S_{\left[\rho_{0}\right]}\right), \mathbf{M}_{\mathcal{N}}\left(\mathrm{O}_{23}, S_{\left[\rho_{0}\right]}\right)$ and $\mathbf{M}_{\mathcal{N}}\left(\mathrm{O}_{24}, S_{\left[\rho_{0}\right]}\right)$. And further, we have Bell's inequality in quantum language as follows.

$$
\left|R_{13}-R_{14}\right|+\left|R_{23}+R_{24}\right| \leq 2
$$

Proof. Clearly we see, $i=1,2, j=3,4$,

$$
R_{i j}=\sum_{\left(x_{1}, x_{2}, x_{3}, x_{4}\right) \in X \times X \times X \times X} x_{i} \cdot x_{j} \rho_{0}\left(F\left(\left\{\left(x_{1}, x_{2}, x_{3}, x_{4}\right)\right\}\right)\right)
$$

(for example, $R_{13}=\sum_{\left(x_{1}, x_{2}, x_{3}, x_{4}\right) \in X \times X \times X \times X} x_{1} \cdot x_{3} \rho_{0}\left(F\left(\left\{\left(x_{1}, x_{2}, x_{3}, x_{4}\right)\right\}\right)\right)$ ). Therefore, we see that

$$
\begin{aligned}
& \left|R_{13}-R_{14}\right|+\left|R_{23}+R_{24}\right| \\
& =\sum_{\left(x_{1}, x_{2}, x_{3}, x_{4}\right) \in X \times X \times X \times X}\left[\left|x_{1} \cdot x_{3}-x_{1} \cdot x_{4}\right|+\left|x_{2} \cdot x_{3}+x_{2} \cdot x_{4}\right|\right] \rho_{0}\left(F\left(\left\{\left(x_{1}, x_{2}, x_{3}, x_{4}\right)\right\}\right)\right) \\
& =\sum_{\left(x_{1}, x_{2}, x_{3}, x_{4}\right) \in X \times X \times X \times X}\left[\left|x_{3}-x_{4}\right|+\left|x_{3}+x_{4}\right|\right] \rho_{0}\left(F\left(\left\{\left(x_{1}, x_{2}, x_{3}, x_{4}\right)\right\}\right)\right) \leq 2
\end{aligned}
$$

This completes the proof.

As the corollary of this theorem, we have the followings:

Corollary 7. Consider the parallel measurement

$\otimes_{i=1,2, j=3,4} \mathrm{M}_{\mathcal{N}}\left(\mathrm{O}_{i j}=\left(X^{2}, \mathcal{P}\left(X^{2}\right), F_{i j}\right), S_{\left[\rho_{0}\right]}\right)$ as in Theorem 6. Let

$$
x=\left(\left(x_{13}^{1}, x_{13}^{2}\right),\left(x_{14}^{1}, x_{14}^{2}\right),\left(x_{23}^{1}, x_{23}^{2}\right),\left(x_{24}^{1}, x_{24}^{2}\right)\right) \in X^{8}\left(\equiv\{-1,1\}^{8}\right)
$$

be a measured value of the parallel measurement $\otimes_{i=1,2, j=3,4} \mathrm{M}_{\mathcal{N}}\left(\mathrm{O}_{i j}=\left(X^{2}, \mathcal{P}\left(X^{2}\right), F_{i j}\right), S_{\left[\rho_{0}\right]}\right)$. Let $N$ be sufficiently large natural number. Consider $N$-parallel measurement $\bigotimes_{n=1}^{N}\left[\otimes_{i=1,2, j=2,3} \mathrm{M}_{\mathcal{N}}\left(\mathrm{O}_{i j}=\left(X^{2}, \mathcal{P}\left(X^{2}\right), F_{i j}\right), S_{\left[\rho_{0}\right]}\right)\right]$. Let $\left\{x^{n}\right\}_{n=1}^{N}$ be the measured value. That is,

$$
\left\{x^{n}\right\}_{n=1}^{N}=\left[\begin{array}{c}
\left(\left(x_{13}^{1,1}, x_{13}^{2,1}\right),\left(x_{14}^{1,1}, x_{14}^{2,1}\right),\left(x_{23}^{1,1}, x_{23}^{2,1}\right),\left(x_{24}^{1,1}, x_{24}^{2,1}\right)\right) \\
\left(\left(x_{13}^{1,2}, x_{13}^{2,2}\right),\left(x_{14}^{1,2}, x_{14}^{2,2}\right),\left(x_{23}^{1,2}, x_{23}^{2,2}\right),\left(x_{24}^{1,2}, x_{24}^{2,2}\right)\right) \\
\vdots \\
\left(\left(x_{13}^{1, N}, x_{13}^{2, N}\right),\left(x_{14}^{1, N}, x_{14}^{2, N}\right),\left(x_{23}^{1, N}, x_{23}^{2, N}\right),\left(x_{24}^{1, N}, x_{24}^{2, N}\right)\right)
\end{array}\right] \in\left(X^{8}\right)^{N}
$$

Here, note that the law of large numbers says: for sufficiently large $N$,

$$
R_{i j} \approx \frac{1}{N} \sum_{n=1}^{N} x_{i j}^{1, n} x_{i j}^{2, n} \quad(i=1,2, j=3,4)
$$

Then, it holds, by the Formula (5), that 


$$
\left|\sum_{n=1}^{N} \frac{x_{13}^{1, n} x_{13}^{2, n}}{N}-\sum_{n=1}^{N} \frac{x_{14}^{1, n} x_{14}^{2, n}}{N}\right|+\left|\sum_{n=1}^{N} \frac{x_{23}^{1, n} x_{23}^{2, n}}{N}+\sum_{n=1}^{N} \frac{x_{24}^{1, n} x_{24}^{2, n}}{N}\right| \leq 2,
$$

which is also called Bell's inequality in quantum language.

Remark 8. [The conventional Bell's inequality ( $c f$. refs. [17] [16] [18])] The mathematical Bell's inequality is as follows: Let $(\Theta, \mathcal{B}, P)$ be a probability space. Let $\left(f_{1}, f_{2}, f_{3}, f_{4}\right): \Theta \rightarrow X^{4}\left(\equiv\{-1,1\}^{4}\right)$ be a measurable functions. Define the correlation functions $\hat{R}_{i j}(i=1,2, j=3,4)$ by $\int_{\Theta} f_{i}(\theta) f_{j}(\theta) P(\mathrm{~d} \theta)$. Then, the following mathematical Bell's inequality (or precisely, CHSH inequality (cf. ref. [16])) holds:

$$
\left|\tilde{R}_{13}-\tilde{R}_{14}\right|+\left|\tilde{R}_{23}+\tilde{R}_{24}\right| \leq 2
$$

(E) This is easily proved as follows.

"the left-hand side of the above (8)"

$$
\leq \int_{\Theta}\left|f_{3}(\theta)-f_{4}(\theta)\right| P(\mathrm{~d} \theta)+\int_{\Theta}\left|f_{3}(\theta)+f_{4}(\theta)\right| P(\mathrm{~d} \theta) \leq 2
$$

This completes the proof.

Recall Theorem 6 (Bell's inequality in quantum language), in which we have, by the combinable condition, the probability space $\left(X^{4}, \mathcal{P}\left(X^{4}\right), \rho_{0}(F(\cdot))\right)$. Therefore the proof of Theorem 6 and the above proof $(\mathrm{E})$ are, from the mathematical point of view, the same.

\section{3. “Bell's Inequality” Is Violated in Classical Systems as Well as Quantum Systems}

In the previous section, we show that Theorem 6 (or Corollary 7) says

$\left(\mathrm{F}_{1}\right)$ Under the combinable condition (cf. Definition 4), Bell's Inequality (5) (or, (7)) holds in both classical systems and quantum systems.

Or, equivalently,

$\left(\mathrm{F}_{2}\right)$ If Bell's Inequality (5) (or (7)) is violated, then the combined observable does not exist, and thus, we cannot obtain the measured value (by the combined measurement).

This is similar to the following elementary statement in quantum mechanics:

$\left(\mathrm{F}_{2}^{\prime}\right)$ We have no (generalized) simultaneous measurement of the position observable $Q$ and the momentum observable $P$, and thus we cannot obtain the measured value (by the generalized simultaneous measurement),

which may be, from Einstein's point of view, represented that "true value (or, hidden variable) of the position and momentum" does not exist. Since the error $\Delta$ is usually defined by $\Delta=$ rough measured value - true value , it is not easy to define the errors $\Delta_{Q}$ and $\Delta_{P}$ in Heisenberg's uncertainty principle $\Delta_{Q} \cdot \Delta_{P} \geq \hbar / 2$.

This definition was completed and Heisenberg's uncertainty principle was proved in ref. [11]. Also, according to the maxim of dualism: "To be is to be perceived" due to G. Berkeley, we think that it is not necessary to name that does not exist (or equivalently, that is not measured).

The above statement $\left(\mathrm{F}_{2}\right)$ makes us expect that

(G) Bell's inequality (5) (or (7)) is violated in classical systems as well as quan- 
tum systems without the combinable condition.

This $(G)$ was already shown in my previous paper [2]. However, I received a lot of questions concerning $(\mathrm{G})$ from the readers. Thus, in this section, we again explain the $(\mathrm{G})$ precisely.

\section{Bell Test Experiment}

In order to show the $(G)$, three steps ([Step: I] - [Step: III]) are prepared in what follows.

[Step: I].

Put $X=\{-1,1\}$. Define complex numbers $a_{k}\left(=\alpha_{k}+\beta_{k} \sqrt{-1} \in \mathbb{C}:\right.$ the complex field) $(k=1,2,3,4)$ such that $\left|a_{k}\right|=1$. Define the probability space $\left(X^{2}, \mathcal{P}\left(X^{2}\right), v_{a_{i} a_{j}}\right)$ such that $(i=1,2, j=3,4)$

$$
\begin{aligned}
& v_{a_{i} a_{j}}(\{(1,1)\})=v_{a_{i} a_{j}}(\{(-1,-1)\})=\left(1-\alpha_{i} \alpha_{j}-\beta_{i} \beta_{j}\right) / 4 \\
& v_{a_{i} a_{j}}(\{(-1,1)\})=v_{a_{i} a_{j}}(\{(1,-1)\})=\left(1+\alpha_{i} \alpha_{j}+\beta_{i} \beta_{j}\right) / 4
\end{aligned}
$$

The correlation $R\left(a_{i}, a_{j}\right) \quad(i=1,2, j=3,4)$ is defined as follows:

$$
R\left(a_{i}, a_{j}\right) \equiv \sum_{\left(x_{1}, x_{2}\right) \in X \times X} x_{1} \cdot x_{2} v_{a_{i} a_{j}}\left(\left\{\left(x_{1}, x_{2}\right)\right\}\right)=-\alpha_{i} \alpha_{j}-\beta_{i} \beta_{j}
$$

Now we have the following problem:

(H) Find a measurement $\mathrm{M}_{\mathcal{N}}\left(\mathrm{O}_{a_{i} a_{j}}=\left(X^{2}, \mathcal{P}\left(X^{2}\right), F_{a_{i} a_{j}}\right), S_{\left[\rho_{0}\right]}\right)$ $(i=1,2, j=3,4)$ such that

$$
\rho_{0}\left(F_{a_{i} a_{j}}(\Xi)\right)=v_{a_{i} a_{j}}(\Xi) \quad\left(\forall \Xi \in \mathcal{P}\left(X^{2}\right)\right)
$$

and

$$
\begin{aligned}
& F_{a_{1} a_{3}}\left(\left\{x_{1}\right\} \times X\right)=F_{a_{1} a_{4}}\left(\left\{x_{1}\right\} \times X\right), \quad F_{a_{2} a_{3}}\left(\left\{x_{2}\right\} \times X\right)=F_{a_{2} a_{4}}\left(\left\{x_{2}\right\} \times X\right) \\
& F_{a_{1} a_{3}}\left(X \times\left\{x_{3}\right\}\right)=F_{a_{2} a_{3}}\left(X \times\left\{x_{3}\right\}\right), \quad F_{a_{1} a_{4}}\left(X \times\left\{x_{4}\right\}\right)=F_{a_{2} a_{4}}\left(X \times\left\{x_{4}\right\}\right) \\
& \left(\forall x_{k} \in X(\equiv\{-1,1\}), k=1,2,3,4\right)
\end{aligned}
$$

which is the same as the condition in Remark 5.

[Step: II].

Let us answer this problem $(\mathrm{H})$ in the two cases (i.e., classical case and quantum case), that is,

$$
\text { - }\left\{\begin{array}{l}
1 \text { the case of quantum systems : } \\
{\left[\mathcal{A}=B\left(\mathbb{C}^{2}\right) \otimes B\left(\mathbb{C}^{2}\right)\left(\equiv B\left(\mathbb{C}^{2} \otimes \mathbb{C}^{2}\right)\right), \mathcal{N}=B\left(\mathbb{C}^{2}\right) \otimes B\left(\mathbb{C}^{2}\right)\right]} \\
\text { 2) the case of classical systems : } \\
{\left[\mathcal{A}=C_{0}(\Omega) \otimes C_{0}(\Omega)\left(\equiv C_{0}(\Omega \times \Omega)\right), \mathcal{N}=L^{\infty}(\Omega) \otimes L^{\infty}(\Omega)\right]}
\end{array}\right.
$$

1) the case of quantum system: $\left[\mathcal{A}=B\left(\mathbb{C}^{2}\right) \otimes B\left(\mathbb{C}^{2}\right)\right]$ Put

$$
e_{1}=\left[\begin{array}{l}
1 \\
0
\end{array}\right], \quad e_{2}=\left[\begin{array}{l}
0 \\
1
\end{array}\right] \quad\left(\in \mathbb{C}^{2}\right)
$$


For each $a_{k}(k=1,2,3,4)$, define the observable $\mathrm{O}_{a_{k}} \equiv\left(X, \mathcal{P}(X), G_{a_{k}}\right)$ in $B\left(\mathbb{C}^{2}\right)$ such that

$$
G_{a_{k}}(\{1\})=\frac{1}{2}\left[\begin{array}{cc}
1 & \bar{a}_{k} \\
a_{k} & 1
\end{array}\right], G_{a_{k}}(\{-1\})=\frac{1}{2}\left[\begin{array}{cc}
1 & -\bar{a}_{k} \\
-a_{k} & 1
\end{array}\right]
$$

where $\bar{a}_{k}=\alpha_{k}-\beta_{k} \sqrt{-1}$. Then, we have four observable:

$$
\hat{\mathrm{O}}_{a_{i}}=\left(X, \mathcal{P}(X), G_{a_{i}} \otimes I\right), \quad \hat{\mathrm{O}}_{a_{j}}=\left(X, \mathcal{P}(X), I \otimes G_{a_{j}}\right) \quad(i=1,2, j=3,4)
$$

and further,

$$
\mathrm{O}_{a_{i} a_{j}}=\left(X^{2}, \mathcal{P}\left(X^{2}\right), F_{a_{i} a_{j}}:=G_{a_{i}} \otimes G_{a_{j}}\right) \quad(i=1,2, j=3,4)
$$

in $B\left(\mathbb{C}^{2}\right) \otimes B\left(\mathbb{C}^{2}\right)$, where it should be noted that $F_{a_{i} a_{j}}$ is separated by $G_{a_{i}}$ and $G_{a_{j}}$.

Further define the singlet state $\rho_{0}=\left|\psi_{s}\right\rangle\left\langle\psi_{s}\right|\left(\in S^{p}\left(B\left(\mathbb{C}^{2} \otimes \mathbb{C}^{2}\right)^{*}\right)\right)$, where

$$
\psi_{s}=\left(e_{1} \otimes e_{2}-e_{2} \otimes e_{1}\right) / \sqrt{2}
$$

Thus we have the measurement $\mathrm{M}_{B\left(\mathbb{C}^{2} \otimes \mathbb{C}^{2}\right)^{*}}\left(\mathrm{O}_{a_{i} a_{j}}, S_{\left[\rho_{0}\right]}\right)$ in $B\left(\mathbb{C}^{2}\right) \otimes B\left(\mathbb{C}^{2}\right)$ $(i=1,2, j=3,4)$. The followings are clear: for each $\left(x_{1}, x_{2}\right) \in X^{2}\left(\equiv\{-1,1\}^{2}\right)$,

$$
\begin{aligned}
\rho_{0}\left(F_{a_{i} a_{j}}\left(\left\{\left(x_{1}, x_{2}\right)\right\}\right)\right) & =\left\langle\psi_{s},\left(G_{a_{i}}\left(\left\{x_{1}\right\}\right) \otimes G_{a_{j}}\left(\left\{x_{2}\right\}\right)\right) \psi_{s}\right\rangle \\
& =v_{a_{i} a_{j}}\left(\left\{\left(x_{1}, x_{2}\right)\right\}\right) \quad(i=1,2, j=3,4)
\end{aligned}
$$

For example, we easily see:

$$
\begin{aligned}
& \rho_{0}\left(F_{a_{i} b_{j}}(\{(1,1)\})\right)=\left\langle\psi_{s},\left(G_{a_{i}}(\{1\}) \otimes G_{a_{j}}(\{1\})\right) \psi_{s}\right\rangle \\
= & \frac{1}{8}\left\langle\left(e_{1} \otimes e_{2}-e_{2} \otimes e_{1}\right),\left(\left[\begin{array}{cc}
1 & \bar{a}_{i} \\
a_{i} & 1
\end{array}\right] \otimes\left[\begin{array}{cc}
1 & \bar{a}_{j} \\
a_{j} & 1
\end{array}\right]\right)\left(e_{1} \otimes e_{2}-e_{2} \otimes e_{1}\right)\right\rangle \\
= & \frac{1}{8}\left\langle\left(\left[\begin{array}{l}
1 \\
0
\end{array}\right] \otimes\left[\begin{array}{l}
0 \\
1
\end{array}\right]-\left[\begin{array}{l}
0 \\
1
\end{array}\right] \otimes\left[\begin{array}{l}
1 \\
0
\end{array}\right]\right),\left(\left[\begin{array}{cc}
1 & \bar{a}_{i} \\
a_{i} & 1
\end{array}\right] \otimes\left[\begin{array}{cc}
1 & \bar{a}_{j} \\
a_{j} & 1
\end{array}\right]\right)\left(\left[\begin{array}{l}
1 \\
0
\end{array}\right] \otimes\left[\begin{array}{l}
0 \\
1
\end{array}\right]-\left[\begin{array}{l}
0 \\
1
\end{array}\right] \otimes\left[\begin{array}{l}
1 \\
0
\end{array}\right]\right)\right\rangle \\
= & \frac{1}{8}\left\langle\left(\left[\begin{array}{l}
1 \\
0
\end{array}\right] \otimes\left[\begin{array}{l}
0 \\
1
\end{array}\right]-\left[\begin{array}{l}
0 \\
1
\end{array}\right] \otimes\left[\begin{array}{l}
1 \\
0
\end{array}\right]\right),\left(\left[\begin{array}{c}
1 \\
a_{i}
\end{array}\right] \otimes\left[\begin{array}{c}
\bar{a}_{j} \\
1
\end{array}\right]-\left[\begin{array}{c}
\bar{a}_{i} \\
1
\end{array}\right] \otimes\left[\begin{array}{c}
1 \\
a_{j}
\end{array}\right]\right)\right\rangle \\
= & \frac{1}{8}\left(2-\alpha_{i} \bar{a}_{j}-\bar{a}_{i} a_{j}\right)=\left(1-\alpha_{i} \alpha_{j}-\beta_{i} \beta_{j}\right) / 4=v_{a_{i} a_{j}}(\{(1,1)\})
\end{aligned}
$$

Therefore, the measurement $\mathrm{M}_{B\left(\mathbb{C}^{2} \otimes \mathbb{C}^{2}\right)}\left(\mathrm{O}_{a_{i} a_{j}}, S_{\left[\rho_{0}\right]}\right)$ satisfies the condition $(\mathrm{H})$.

2) the case of classical systems: $\left[\mathcal{A}=C_{0}(\Omega) \otimes C_{0}(\Omega)=C_{0}(\Omega \times \Omega)\right]$

Put $\omega_{0}\left(=\left(\omega_{0}^{\prime}, \omega_{0}^{\prime \prime}\right)\right) \in \Omega \times \Omega, \rho_{0}=\delta_{\omega_{0}}\left(\in \mathfrak{S}^{p}\left(C_{0}(\Omega \times \Omega)^{*}\right)\right.$, i.e., the point measure at $\left.\left.\omega_{0}\right)\right)$. Define the observable $\mathrm{O}_{a_{i} a_{j}}:=\left(X^{2}, \mathcal{P}\left(X^{2}\right), F_{a_{i} a_{j}}\right)$ in $L^{\infty}(\Omega \times \Omega)$ such that

$$
\begin{aligned}
& {\left[F_{a_{i} a_{j}}\left(\left\{\left(x_{1}, x_{2}\right)\right\}\right)\right](\omega)=v_{a_{i} a_{j}}\left(\left\{\left(x_{1}, x_{2}\right)\right\}\right)} \\
& \left(\forall\left(x_{1}, x_{2}\right) \in X^{2}, i=1,2, j=3,4, \forall \omega \in \Omega \times \Omega\right)
\end{aligned}
$$

Thus, we have four observables 


$$
\mathrm{O}_{a_{i} a_{j}}=\left(X^{2}, \mathcal{P}\left(X^{2}\right), F_{a_{i} a_{j}}\right) \quad(i=1,2, j=3,4)
$$

in $L^{\infty}(\Omega \times \Omega)$ (though the variables are not separable (cf. the formula (13)). Then, it is clear that the measurement $\mathrm{M}_{C_{0}(\Omega \times \Omega)}\left(\mathrm{O}_{a_{i} a_{j}}, S_{\left[\delta_{\omega_{0}}\right]}\right)$ satisfies the condition $(\mathrm{H})$.

2)' the case of classical systems: $\left[\mathcal{A}=C_{0}(\Omega) \otimes C_{0}(\Omega)=C_{0}(\Omega \times \Omega)\right]$

It is easy to show a lot of different answers from the above 2 ). For example, as a slight generalization of (9), define the probability measure $v_{a_{i} a_{j}}^{t}(0 \leq t \leq 1)$ such that

$$
\begin{aligned}
& v_{a_{i} a_{j}}^{t}(\{(1,1)\})=v_{a_{i} a_{j}}^{t}(\{(-1,-1)\})=\left(1-t\left(\alpha_{i} \alpha_{j}+\beta_{i} \beta_{j}\right)\right) / 4 \\
& v_{a_{i} a_{j}}^{t}(\{(-1,1)\})=v_{a_{i} a_{j}}^{t}(\{(1,-1)\})=\left(1+t\left(\alpha_{i} \alpha_{j}+\beta_{i} \beta_{j}\right)\right) / 4
\end{aligned}
$$

And consider the real-valued continuous function $t\left(\in C_{0}(\Omega \times \Omega)\right)$ such that $0 \leq t\left(\omega^{\prime}, \omega^{\prime \prime}\right) \leq 1 \quad\left(\forall \omega=\left(\omega^{\prime}, \omega^{\prime \prime}\right) \in \Omega \times \Omega\right)$. And assume that $t\left(\omega_{0}\right)=1$ for some $\omega_{0}\left(=\left(\omega_{0}^{\prime}, \omega_{0}^{\prime \prime}\right)\right) \in \Omega \times \Omega, \rho_{0}=\delta_{\omega_{0}}\left(\in \mathfrak{S}^{p}\left(C_{0}(\Omega \times \Omega)^{*}\right)\right.$, i.e., the point measure at $\left.\left.\omega_{0}\right)\right)$. Define the observable $\mathrm{O}_{a_{i} a_{j}}:=\left(X^{2}, \mathcal{P}\left(X^{2}\right), F_{a_{i} a_{j}}\right)$ in $L^{\infty}(\Omega \times \Omega)$ such that

$$
\begin{aligned}
& {\left[F_{a_{i} a_{j}}\left(\left\{\left(x_{1}, x_{2}\right)\right\}\right)\right](\omega)=v_{a_{i} a_{j}}^{t(\omega)}\left(\left\{\left(x_{1}, x_{2}\right)\right\}\right)} \\
& \left(\forall\left(x_{1}, x_{2}\right) \in X^{2}, i=1,2, j=3,4, \forall \omega \in \Omega \times \Omega\right)
\end{aligned}
$$

Thus, we have four observables

$$
\mathrm{O}_{a_{i} a_{j}}=\left(X^{2}, \mathcal{P}\left(X^{2}\right), F_{a_{i} a_{j}}\right) \quad(i=1,2, j=3,4)
$$

in $L^{\infty}(\Omega \times \Omega)$ (though the variables are not separable (cf. the Formula (13)). Then, it is clear that the measurement $\mathrm{M}_{L^{\infty}(\Omega \times \Omega)}\left(\mathrm{O}_{a_{i} a_{j}}, S_{\left[\delta_{\infty 0}\right]}\right)$ satisfies the condition $(\mathrm{H})$.

[Step: III].

As defined by (9), consider four complex numbers $a_{k}\left(=\alpha_{k}+\beta_{k} \sqrt{-1} ; k=1,2,3,4\right)$ such that $\left|a_{k}\right|=1$. Thus we have four observables

$$
\begin{array}{cc}
\mathrm{O}_{a_{1} a_{3}}:=\left(X^{2}, \mathcal{P}\left(X^{2}\right), F_{a_{1} a_{3}}\right), & \mathrm{O}_{a_{1} a_{4}}:=\left(X^{2}, \mathcal{P}\left(X^{2}\right), F_{a_{1} a_{4}}\right), \\
\mathrm{O}_{a_{2} a_{3}}:=\left(X^{2}, \mathcal{P}\left(X^{2}\right), F_{a_{2} a_{3}}\right), & \mathrm{O}_{a_{2} a_{4}}:=\left(X^{2}, \mathcal{P}\left(X^{2}\right), F_{a_{2} a_{4}}\right),
\end{array}
$$

in $\mathcal{N}$. Thus, we have the parallel measurement $\otimes_{i=1,2, j=3,4} \mathrm{M}_{\mathcal{N}}\left(\mathrm{O}_{a_{i} a_{j}}:=\left(X^{2}, \mathcal{P}\left(X^{2}\right), F_{a_{i} a_{j}}\right), S_{\left[\rho_{0}\right]}\right)$ in $\otimes_{i=1,2, j=3,4} \mathcal{N}$.

Thus, putting

$$
a_{1}=\sqrt{-1}, a_{2}=1, a_{3}=\frac{1+\sqrt{-1}}{\sqrt{2}}, a_{4}=\frac{1-\sqrt{-1}}{\sqrt{2}},
$$

we see, by (10), that

$$
\left|R\left(a_{1}, a_{3}\right)-R\left(a_{1}, a_{4}\right)\right|+\left|R\left(a_{2}, a_{3}\right)+R\left(a_{2}, a_{4}\right)\right|=2 \sqrt{2}
$$

Further, assume that the measured value is $x\left(\in X^{8}\right)$. That is,

$$
x=\left(\left(x_{13}^{1}, x_{13}^{2}\right),\left(x_{14}^{1}, x_{14}^{2}\right),\left(x_{23}^{1}, x_{23}^{2}\right),\left(x_{24}^{1}, x_{24}^{2}\right)\right) \in \underset{i, j=1,2}{\times} X^{2}\left(\equiv\{-1,1\}^{8}\right)
$$


Let $N$ be sufficiently large natural number. Consider $N$-parallel measurement $\bigotimes_{n=1}^{N}\left[\bigotimes_{i=1,2, j=3,4} \mathrm{M}_{\mathcal{N}}\left(\mathrm{O}_{a_{i} a_{j}}:=\left(X^{2}, \mathcal{P}\left(X^{2}\right), F_{a_{i} a_{j}}\right), S_{\left[\rho_{0}\right]}\right)\right]$. Assume that its measured value is $\left\{x^{n}\right\}_{n=1}^{N}$. That is,

$$
\begin{aligned}
\left\{x^{n}\right\}_{n=1}^{N}= & {\left[\begin{array}{c}
\left(\left(x_{13}^{1,1}, x_{13}^{2,1}\right),\left(x_{14}^{1,1}, x_{14}^{2,1}\right),\left(x_{23}^{1,1}, x_{23}^{2,1}\right),\left(x_{24}^{1,1}, x_{24}^{2,1}\right)\right) \\
\left(\left(x_{13}^{1,2}, x_{13}^{2,2}\right),\left(x_{14}^{1,2}, x_{14}^{2,2}\right),\left(x_{23}^{1,2}, x_{23}^{2,2}\right),\left(x_{24}^{1,2}, x_{24}^{2,2}\right)\right) \\
\vdots \\
\left(\left(x_{13}^{1, N}, x_{13}^{2, N}\right),\left(x_{14}^{1, N}, x_{14}^{2, N}\right),\left(x_{23}^{1, N}, x_{23}^{2, N}\right),\left(x_{24}^{1, N}, x_{24}^{2, N}\right)\right)
\end{array}\right] } \\
& \in\left(\underset{i=1,2, j=3,4}{x} X^{2}\right)^{N}\left(\equiv\{-1,1\}^{8 N}\right)
\end{aligned}
$$

Then, the law of large numbers says that

$$
R\left(a_{i}, a_{j}\right) \approx \frac{1}{N} \sum_{n=1}^{N} x_{i j}^{1, n} x_{i j}^{2, n} \quad(i=1,2, j=3,4)
$$

This and the Formula (18) say that

$$
\left|\sum_{n=1}^{N} \frac{x_{13}^{1, n} x_{13}^{2, n}}{N}-\sum_{n=1}^{N} \frac{x_{14}^{1, n} x_{14}^{2, n}}{N}\right|+\left|\sum_{n=1}^{N} \frac{x_{23}^{1, n} x_{23}^{2, n}}{N}+\sum_{n=1}^{N} \frac{x_{24}^{1, n} x_{24}^{2, n}}{N}\right| \approx 2 \sqrt{2}
$$

Therefore, Bell's Inequality (5) (or (7)) is violated in classical systems as well as quantum systems.

Remark 9. For completeness, note that the observables $\mathrm{O}_{a_{i} a_{j}} \quad(i=1,2, j=3,4)$ in the classical $L^{\infty}(\Omega \times \Omega)$ are not combinable in spite that these commute. Also, note that the Formulas (16) and (17) imply that

$$
\begin{gathered}
{\left[F_{a_{1} a_{3}}(\{x\} \times X)\right](\omega)=\left[F_{a_{1} a_{4}}(\{x\} \times X)\right](\omega)=1 / 2,} \\
{\left[F_{a_{2} a_{3}}(\{x\} \times X)\right](\omega)=\left[F_{a_{2} a_{4}}(\{x\} \times X)\right](\omega)=1 / 2,} \\
{\left[F_{a_{1} a_{3}}(X \times\{x\})\right](\omega)=\left[F_{a_{2} a_{3}}(X \times\{x\})\right](\omega)=1 / 2,} \\
{\left[F_{a_{1} a_{4}}(X \times\{x\})\right](\omega)=\left[F_{a_{2} a_{4}}(X \times\{x\})\right](\omega)=1 / 2} \\
(\forall x \in X, \forall \omega \in \Omega \times \Omega),
\end{gathered}
$$

which is similar as in Remark $5 ; 1)$ or in $(\mathrm{H})$.

\section{Conclusions}

In Bohr-Einstein debates (refs. [13] [14]), Einstein's standing-point (that is, "the moon is there whether one looks at it or not" (i.e., physics holds without observers)) is on the side of the realistic world view in Figure 1. On the other hand, we think that Bohr's standing point (that is, "to be is to be perceived" (i.e., there is no science without measurements)) is on the side of the linguistic world view in Figure 1.

In this paper, contrary to Bell's spirit (which inherits Einstein's spirit), we try to discuss Bell's inequality in Bohr's spirit (i.e., in the framework of quantum lan- 
guage). And we show Theorem 6 (Bell's inequality in quantum language), which says the statement $\left(\mathrm{F}_{2}\right)$, that is,

$\left(\mathrm{I}_{1}\right)\left(\equiv\left(\mathrm{F}_{2}\right)\right)$ : If Bell's Inequality (5) (or (7)) is violated, then the combined observable does not exist, and thus, we cannot obtain the measured value (by the measurement of the combined observable).

Also, recall that Bell's original argument says, roughly speaking, that

$\left(\mathrm{I}_{2}\right)$ If the mathematical Bell's Inequality (8) is violated in Bell test experiment (the quantum case of Section 3.1), then hidden variables do not exist.

It should be note that the concept of "hidden variable" is independent of measurements, thus, the $\left(\mathrm{I}_{2}\right)$ is a philosophical statement in Einstein's spirit, on the other hand, the $\left(\mathrm{I}_{1}\right)$ is a statement in Bohr's spirit (i.e., there is no science without measurements). It is sure that Bell's answer $\left(\mathrm{I}_{2}\right)$ is attractive philosophically, however, we believe in the scientific superiority of our answer $\left(\mathrm{I}_{1}\right)$. That is, we think that our $\left(I_{1}\right)$ is a scientific representation of the philosophical $\left(I_{2}\right)$. If so, we can, for the first time, understand Bell's inequality in science. That is, Theorem 6 is the true Bell's inequality. And we conclude that whether or not Bell's inequality holds does not depend on whether classical systems or quantum systems (in Section 3), but depend on whether the combined measurement exists or not (in Section 2).

We hope that our proposal will be examined from various points of view ${ }^{1}$.

\section{References}

[1] Ishikawa, S. (2006) Mathematical Foundations of Measurement Theory. Keio University Press Inc. Tokyo, 335 p. http://www.keio-up.co.jp/kup/mfomt/

[2] Ishikawa, S. (2011) A New Interpretation of Quantum Mechanics. Journal of Quantum Information Science, 1, 35-42.

http://www.scirp.org/journal/PaperInformation.aspx?paperID=7610 https://doi.org/10.4236/jqis.2011.12005

[3] Ishikawa, S. (2012) Quantum Mechanics and the Philosophy of Language: Reconsideration of Traditional Philosophies. Journal of Quantum Information Science, 2, 2-9. http://www.scirp.org/journal/PaperInformation.aspx?paperID $=18194$ https://doi.org/10.4236/jqis.2012.21002

[4] Ishikawa, S. (2012) A Measurement Theoretical Foundation of Statistics. Applied Mathematics, 3, 283-292.

http://www.scirp.org/Journal/PaperInformation.aspx?PaperID=18109 https://doi.org/10.4236/am.2012.33044

[5] Ishikawa, S. (2012) Monty Hall Problem and the Principle of Equal Probability in Measurement Theory. Applied Mathematics, 3, 788-794.

http://www.scirp.org/journal/PaperInformation.aspx?PaperID=19884 https://doi.org/10.4236/am.2012.37117

[6] Ishikawa, S. (2012) Ergodic Hypothesis and Equilibrium Statistical Mechanics in the Quantum Mechanical World View. World Journal of Mechanics, 2, 125-130. http://www.scirp.org/journal/PaperInformation.aspx?PaperID=18861 https://doi.org/10.4236/wjm.2012.22014

[7] Ishikawa, S. (2015) Linguistic Interpretation of Quantum Mechanics; Projection ${ }^{1}$ For the further information of quantum language, see http://www.math.keio.ac.jp/ $\sim$ ishikawa/indexe.html 
Postulate. Journal of Quantum Information Science, 5, 150-155.

http://www.scirp.org/Journal/PaperInformation.aspx?PaperID=62464 https://doi.org/10.4236/jqis.2015.54017

[8] Ishikawa, S. (2016) Linguistic Interpretation of Quantum Mechanics: Towards World-Description in Quantum Language. Shiho-Shuppan Publisher, Tsukuba, 416 p. http://www.math.keio.ac.jp/academic/research_pdf/report/2016/16001.pdf

[9] Ishikawa, S. (2017) A Final Solution to Mind-Body Problem by Quantum Language. Journal of Quantum Information Science, 7, 48-56.

http://www.scirp.org/Journal/PaperInformation.aspx?PaperID=76391 https://doi.org/10.4236/jqis.2017.72005

[10] Ishikawa, S. (2017) Bell's Inequality Is Violated in Classical Systems as Well as Quantum Systems. The $W^{*}$-Algebraic Version of This Paper, Research Report, Keio University, Yokohama, $10 \mathrm{p}$. http://www.math.keio.ac.jp/academic/research_pdf/report/2017/17006.pdf

[11] Ishikawa, S. (1991) Uncertainty Relation in Simultaneous Measurements for Arbitrary Observables. Reports on Mathematical Physics, 9, 257-273.

http://www.sciencedirect.com/science/article/pii/003448779190046P https://doi.org/10.1016/0034-4877(91)90046-P

[12] Ishikawa, S. (1997) Fuzzy Inferences by Algebraic Method. Fuzzy Sets and Systems, 87, 181-200. http://www.sciencedirect.com/science/article/pii/S0165011496000358 https://doi.org/10.1016/S0165-0114(96)00035-8

[13] Einstein, A., Podolosky, B. and Rosen, N. (1935) Can Quantum-Mechanical Description of Reality Be Considered Completely? Physical Review, 47, 777-780. https://doi.org/10.1103/PhysRev.47.777

[14] Bohr, N. (1935) Can Quantum-Mechanical Description of Physical Reality Be Considered Complete? Physical Review, 48, 696-702. https://doi.org/10.1103/PhysRev.48.696

[15] Bell, J.S. (1964) On the Einstein-Podolosky-Rosen Paradox. Physics, 1, 195-200.

[16] Clauser, J.F., Horne, M.A., Shimony, A. and Holt, R.A. (1969) Proposed Experiment to Test Local Hidden Variable Theories. Physical Review Letters, 23, 880-884. https://doi.org/10.1103/PhysRevLett.23.880

[17] Redhead, M. (1987) Incompleteness, Non-Locality, and Realism. Oxford University Press, Oxford.

[18] Selleri, F. (1983) Die Debatte um die Quantentheorie, Friedr. Vieweg \& Sohn Verlagsgesellscvhaft $\mathrm{MBH}$, Braunschweig.

[19] Sakai, S. (1971) $C^{*}$-Algebras and $W^{*}$-Algebras. Springer-Verlag, Berlin Heidelberg.

[20] Yosida, K. (1980) Functional Analysis. Springer-Verlag, 6th Edition, Berlin Heidelberg New York.

[21] Davies, E.B. (1976) Quantum Theory of Open Systems. Academic Press, London, New York.

[22] Born, M. (1926) Zur Quantenmechanik der Stoßprozesse (Vorläufige Mitteilung). Zeitschrift für Physik, 37, 863-867. 